\title{
Visual acuity and central retinal thickness: fulfilment of retreatment criteria for recurrent neovascular AMD in routine clinical care
}

\author{
Lukas Reznicek, Johanna Muhr, Michael Ulbig, Anselm Kampik, Wolfgang J Mayer, \\ Christos Haritoglou, Aljoscha Neubauer, Armin Wolf
}

Department of Ophthalmology, Ludwig-Maximilians-University Muenchen, Germany

\section{Correspondence to} Dr Lukas Reznicek, Department of Ophthalmology, Ludwig-Maximilians-University, Klinikum der Universität München, Campus Innenstadt, Mathildenstrasse 8, Munich D-80336, Germany; Lukas.Reznicek@ med.uni-muenchen.de

Received 29 September 2013 Revised 10 April 2014 Accepted 6 May 2014 Published Online First 5 June 2014
CrossMark

To cite: Reznicek L, Muhr J, Ulbig $\mathrm{M}$, et al. Br J Ophthalmol 2014:98: 1333-1337.

\section{ABSTRACT}

Background To evaluate the fulfilment of retreatment criteria in recurrent neovascular age-related macular degeneration (nAMD) for a pro-re-nata treatment regime with ranibizumab in routine clinical care.

Methods Data from patients with treatment-naive nAMD were analysed retrospectively. As an 'upload', all patients had received three-monthly intravitreal ranibizumab injections in a university eye hospital and were then seen by ophthalmologists in private practice who referred them back in case of recurrence. Recurrence was defined as a decrease of visual acuity (VA) of one line or more (functional retreatment criteria), a central retinal thickness (CRT) increase of at least $100 \mu \mathrm{m}$ upon Optical Coherence Tomography (OCT) examination (morphological retreatment criteria) or a new macular haemorrhage (clinical retreatment criteria).

Results We included 92 patients (36 men and 56 women). The mean VA before retreatment of a recurrence was $-0.63 \pm 0.33 \operatorname{logMAR}$ and improved significantly $(p<0.001)$ by $0.10 \pm 0.16 \log$ MAR to $-0.53 \pm 0.28$ logMAR thereafter. Mean CRT before retreatment was $278.07 \pm 87.56 \mu \mathrm{m}$ and decreased significantly $(p<0.001)$ by $71.22 \pm 106.93$ to $206.85 \pm 60.30 \mu \mathrm{m}$. Evaluation of the fulfilment of retreatment criteria revealed functional retreatment criteria in $82.6 \%$ of patients. However, upon re-evaluation of VA using Early Treatment Diabetic Retinopathy Study (ETDRS) charts in the treatment centre, mean decrease of VA was 10 letters as compared with the end of upload therapy. All patients presented an increased CRT when treated for recurrence of nAMD (mean increase $69.47 \mu \mathrm{m}$ ), but the morphological retreatment criteria (CRT increase of $100 \mu \mathrm{m}$ or more) were fulfilled in only $44.4 \%$ of patients upon Spectral Domain OCT (SD-OCT) evaluation in the treatment centre.

Conclusions In a routine clinical care, evaluation of VA using ETDRS charts seems to be more sensitive than Snellen VA testing. Quantitative OCT-based retreatment criteria (eg, increase of CRT of $100 \mu \mathrm{m}$ or more) appear to be not sensitive enough in a clinical setting with referring ophthalmologists.

\section{INTRODUCTION}

Age-related macular degeneration (AMD) is the leading cause of blindness in the elderly population in industrial countries. ${ }^{12}$

Over the years, intravitreal anti-Vascular Endothelial Growth Factor (VEGF) (ranibizumab, pegaptanib, bevacizumab) injections have revolutionised treatment of neovascular AMD (nAMD), which is responsible for the majority of AMD-related visual loss worldwide. ${ }^{3-6}$
Excellent results have been shown for the treatment of choroidal neovascularisation (CNV) due to AMD with ranibizumab. ${ }^{7}$ Various studies (PIER, PrONTO, EXCITE, SUSTAIN, HORIZON, CATT) have evaluated the course of functional (visual acuity (VA)) as well as structural (central retinal thickness (CRT)) parameters along intravitreal anti-VEGF treatment. $^{9-14}$

While the CATT study showed slightly better results for monthly injections as compared with treatment 'as needed', recent data indicate that in routine clinical care, outcome of anti-VEGF treatment 'as needed' remains behind expectation. This is possibly due to a delayed detection of recurrence ${ }^{15-17}$ compared with 'treat and extend' regimes with more promising results. ${ }^{18}$

While in a study setting, testing of VA is performed using Early Treatment Diabetic Retinopathy Study (ETDRS) charts and permanent macular thickness assessment by Optical Coherence Tomography (OCT), this standard does not apply for routine clinical care. The aim of this study was to investigate retreatment criteria in case of recurrence after initial upload treatment in routine clinical care of a university eye hospital which collaborates with referring ophthalmologists in private practice. Additionally, we retrospectively analysed potential factors influencing the development of VA during retreatment.

\section{METHODS}

\section{Patients}

Data from patients from the Department of Ophthalmology, Ludwig-Maximilians University, Munich, were analysed retrospectively. All patients had received a standard 'upload' therapy for so far untreated nAMD and had suffered from recurrence which was retreated according to the then standardised PrONTO scheme.

Inclusion criteria were recurrent nAMD treated with ranibizumab as well as standard 'upload' therapy consisting of at least three injections at monthly intervals. In addition, only patients referred from outclinic ophthalmologists that had been instructed in the then valid retreatment criteria were included in our study.

Exclusion criteria included disorders of the posterior pole other than AMD (such as occlusive vascular diseases), a documented increase in lens opacity, previous glaucoma or cataract surgery between the termination of 'upload' therapy and the patient presenting with a recurrence of wet AMD. 
Table 1 Patients' characteristics

\begin{tabular}{ll}
\hline Baseline characteristics & \\
\hline Number of patients & 92 \\
Age (years) & $77.3 \pm 7.2$ (range 58-89) \\
OD (right eye)/OS (left eye) & $47 / 45$ \\
Sex (male/female) & $36 / 56$ \\
Refraction & \\
$\quad$ Sphere & $1.52 \pm 1.93$ \\
Cylinder & $-1.31 \pm 1.00$ \\
\hline
\end{tabular}

In the treatment centre, nAMD was diagnosed by a retina specialist via a complete ophthalmic examination and confirmed with fluorescein angiography (HRA2/Spectralis, Heidelberg Engineering, Heidelberg, Germany) and optic coherence tomography (Stratus-OCT, Carl Zeiss-Meditec, Dublin, California, USA).

All patients received at least three intravitreal injections at 1-month interval with ranibizumab (Lucentis, $0.5 \mathrm{mg}$ ) during the upload phase. VA based on ETDRS charts, a complete ophthalmic examination and OCT were obtained before treatment and monthly thereafter according to the PrONTO scheme ${ }^{10}$ until the intraretinal or subretinal fluid disappeared upon OCT examination, which marked the end of 'upload' therapy. This treatment was, according to the then valid recommendation of the German Ophthalmologic Society (DOG) as well as the general understanding of pharmacokinetics, considered valid at that time. For further monthly surveillance, patients were sent back to the referring ophthalmologist in private practice who tested for Snellen VA but not with ETDRS charts. When a need for retreatment occurred, those patients were referred back immediately to the hospital. Retreatment criteria valid at the time were based upon the PrONTO study: VA decrease of at least five letters or one Snellen line, a CRT increase of at least $100 \mu \mathrm{m}$ or a new macular haemorrhage. ${ }^{10}$ Upon presentation in the clinic, VA was tested with ETDRS charts and macular assessment using spectral domain OCT.

\section{Statistical analysis}

Data were collected and analysed using SPSS V.17.0 (SPSS Inc, Chicago, Illinois, USA). A $p$ value of $<0.05$ was considered as statistically significant. Since the majority of ophthalmologists in private practice obtained Snellen VA instead of ETDRS VA, all obtained and documented VA results were transferred into $\log$ MAR values.

\section{RESULTS}

In total, we included 92 patients. Baseline characteristics are given in table 1. For 'upload' therapy, all patients needed a mean of 3.6 \pm 1.6 injections compared with a mean of $4.4 \pm 2.1$ injections required for treatment of recurrence.

The period of review beginning with date of 'upload' therapy until the end of recurrence treatment was $447 \pm 195$ days.

\section{Fulfilment of retreatment criteria}

The mean decrease in VA and increase of CRT between the end of the 'upload' therapy and presentation with recurrence was $-0.26 \pm 0.26 \log$ MAR and $99.7 \pm 101.1 \mu \mathrm{m}$, respectively, as measured by ETDRS charts. While VA decrease was one Snellen line at the referring ophthalmologist, this represented a mean decrease of 10 ETDRS letters upon presentation in the hospital. $^{19}$

The functional retreatment parameter (decrease of VA of five ETDRS letters/one line or more) was fulfilled in $82.6 \%$ of patients. However, with a mean decrease of 10 letters as compared with termination of upload treatment, the loss of VA exceeded dramatically the PrONTO criteria for retreatment (five letters).

The quantitative morphological retreatment criteria (increase of CRT of at least $100 \mu \mathrm{m}$ upon OCT examination) were fulfilled in $44.4 \%$ of all patients. Overall, a mean increase of CRT of $69.47 \mu \mathrm{m}$ was observed and $95.7 \%$ of the patients presented an increase of CRT.

\section{Visual acuity}

Mean VA of all patients presenting with treatment naive nAMD before therapy was $-0.62 \pm 0.33 \log$ MAR and improved significantly $(\mathrm{p}<0.001)$ by $0.24 \pm 0.25$ to $-0.37 \pm 0.24 \log$ MAR after 'upload' therapy. The mean VA at the time of presentation at our hospital with a recurrence diagnosed by the referring ophthalmologist was $-0.63 \pm 0.33 \log \mathrm{MAR}$, and improved significantly $(\mathrm{p}<0.001)$ by $0.10 \pm 0.16$ to $-0.53 \pm 0.28 \log$ MAR after retreatment. Mean VA after 'upload' therapy was $-0.37 \pm 0.24$ $\log$ MAR and significantly $(\mathrm{p}<0.001)$ better than mean VA after retreatment with $-0.53 \pm 0.28 \log \mathrm{MAR}$ with a mean loss of VA of $-0.16 \pm 0.21 \operatorname{logMAR}$ after successful treatment of recurrence (see figure 1).

\section{Central retinal thickness}

Measurements of CRT presented the following development: Mean CRT before 'upload' therapy was $290.42 \pm 85.38 \mu \mathrm{m}$ and decreased significantly $(\mathrm{p}<0.001)$ by $81.82 \pm 98.60 \mu \mathrm{m}$ to $208.60 \pm 69.12 \mu \mathrm{m}$ after 'upload' therapy. Mean CRT before retreatment was $278.07 \pm 87.56 \mu \mathrm{m}$ and decreased significantly $(\mathrm{p}<0.001)$ by $71.22 \pm 106.93 \mu \mathrm{m}$ to $206.85 \pm 60.30 \mu \mathrm{m}$ after retreatment. Mean CRT after 'upload' therapy was 208.60 $\pm 69.12 \mu \mathrm{m}$ and similar to $(\mathrm{p}=0.916)$ to mean CRT after retreatment with $206.85 \pm 60.30 \mu \mathrm{m}$ (see figure 2 ).

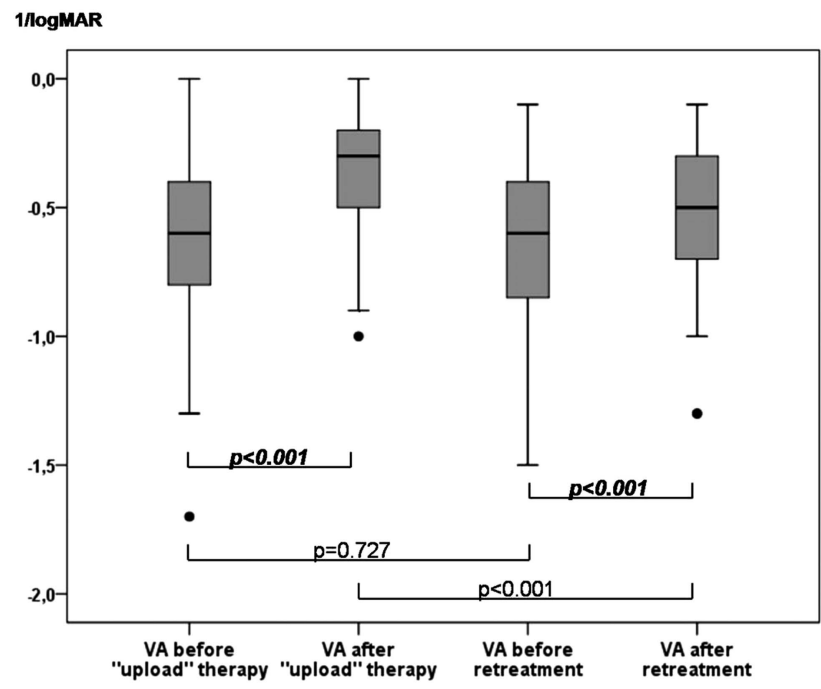

Figure 1 Development of mean visual acuity (VA) in logMAR during observation period before and after 'upload' therapy as well as before and after retreatment of recurrence episode in patients with neovascular age-related macular degeneration. 


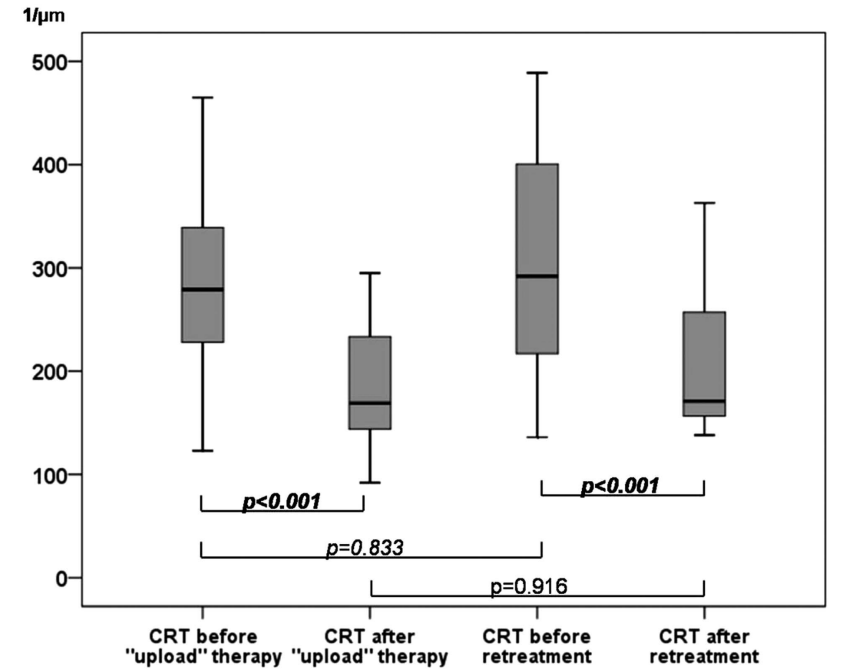

Figure 2 Development of mean central retinal thickness (CRT) during observation period before and after 'upload' therapy as well as before and after retreatment of recurrence episode in patients with neovascular age-related macular degeneration.

\section{Subgroup analysis}

For further evaluation, we subdivided all study eyes into two groups. In the first group ( $n=30$, group 1$)$-which will be referred to as 'recovery'-VA after retreatment of the recurrence episode was not decreased compared with VA after 'upload' therapy, as measured with ETDRS charts. In group 2, $(n=62)$, the 'non-recovery', VA after retreatment was below VA after 'upload' therapy. Both groups did not present significant differences of VA before and after 'upload' therapy (see table 2). However, there was a significant difference between retreatment 'recovery' and 'non-recovery' concerning the VA at the time of presentation with a recurrence: 'non-recovery' showed a significantly lower VA upon recurrence than 'recovery' $(-0.69 \pm 0.31$ vs $-0.50 \pm 0.32 \log$ MAR).

CRT did not differ significantly before and after 'upload' therapy or retreatment (see table 3).

The fulfilment of retreatment criteria in the two groups was as follows:

Group 1: functional retreatment criteria (decrease of VA of five letters (ETDRS-chart) or more) were fulfilled in $56.7 \%$. The morphological retreatment criteria (increase of CRT of $100 \mu \mathrm{m}$ or more) were fulfilled in $41.7 \%$. An increase of CRT, as compared with after 'upload' therapy, was detected in 90.0\% (27 out of 30 eyes) of the patients (mean increase $56.00 \pm 111.85 \mu \mathrm{m}$ ).
Group 2: functional retreatment criteria (decrease of VA of five letters (ETDRS-chart) or more) were fulfilled in 95.2\%. The morphological retreatment criteria (increase of CRT of $100 \mu \mathrm{m}$ or more) were fulfilled in $44.0 \%$. An increase of CRT as compared with after 'upload' therapy was detected in $98.4 \%$ (61 out of 62 eyes) of the patients (mean increase 75.70 $\pm 94.62 \mu \mathrm{m})$.

\section{DISCUSSION}

In this retrospective analysis, both the functional and structural parameters VA and CRT improved significantly in patients with nAMD treated with intravitreal ranibizumab for the initial as well as recurrent episode of $\mathrm{CNV}$ due to AMD. Our findings are in agreement with previously published studies. ${ }^{20}$ While CRT decreased significantly after treatment of the recurrence and was not significantly different from CRT after primary treatment of the initial CNV, VA improved during the treatment of recurrent nAMD but remained significantly worse than after 'upload' therapy $(-0.37 \pm 0.24$ vs $-0.53 \pm 0.28 \log \mathrm{MAR})$ in our clinical routine care.

A progressive damage of the neurosensory retina over the course of the relapsing disease may result in an impairment of retinal function after initial 'upload' therapy. Such an impairment may be more resistant to therapy than the structural parameter 'retinal thickness' caused by macular oedema. ${ }^{21}$ This may be a possible explanation for the moderate difference between structural versus functional outcome after recurrence treatment of nAMD with ranibizumab. Interestingly, the most frequent retreatment criteria in this clinical setting was a loss of five letters or more, while the quantitative OCT retreatment criteria of an increase of $100 \mu \mathrm{m}$ or more were fulfilled in fewer cases. Taking into account a recently published study by Patel and Tufail which showed that most of the retreatment indications during retreatment in the prospective $\mathrm{ABC}$ study were based upon the fulfilment of OCT retreatment criteria, we interpret our observation to mean that obtaining Snellen VA as a functional parameter alone may not be sensitive enough to determine recurrence. $^{22}$

This is of importance as recent studies on routine clinical care situation in Germany (WAVE/COMPASS) show that the use of OCT for detection of recurrences is not widely spread, and only a small number of ophthalmologists $(4.1 \%)$ use OCT for control of macular oedema after anti-VEGF treatment. ${ }^{23}$

The observed mean decrease of VA at the time of presentation, with a recurrence in our hospital, was exceeding the retreatment criteria (five letters, one Snellen line) by far. We consider this may be due to different methods of VA testing. While patients were referred with a decrease of one Snellen

Table 2 Mean visual acuity (VA) in logMAR for group 1 'recovery', group 2 'non-recovery' and their difference before and after 'upload' therapy and retreatment, respectively

\begin{tabular}{|c|c|c|c|c|}
\hline & $\begin{array}{l}\text { Mean VA } \\
\text { group } 1 \text { 'recovery' }\end{array}$ & $\begin{array}{l}\text { Mean VA } \\
\text { group } 2 \text { 'non-recovery' }\end{array}$ & $\begin{array}{l}\text { Difference } \\
\text { group 1-group } 2\end{array}$ & $\begin{array}{l}95 \% \mathrm{Cl} \text { of difference } \\
\text { group 1-group } 2\end{array}$ \\
\hline VA before 'upload' therapy & $-0.67 \pm 0.36$ & $-0.59 \pm 0.31$ & $\begin{array}{l}0.075 \pm 0.077 \\
p=0.337\end{array}$ & -0.080 to 0.230 \\
\hline VA after 'upload' therapy & $-0.41 \pm 0.27$ & $-0.35 \pm 0.23$ & $\begin{array}{l}0.061 \pm 0.057 \\
p=0.337\end{array}$ & -0.053 to 0.177 \\
\hline VA before retreatment & $-0.50 \pm 0.32$ & $-0.69 \pm 0.31$ & $\begin{array}{l}-0.197 \pm 0.071 \\
p=0.007\end{array}$ & -0.338 to -0.055 \\
\hline VA after retreatment & $-0.67 \pm 0.36$ & $-0.40 \pm 0.28$ & $\begin{array}{l}-0.200 \pm 0.061 \\
p=0.002\end{array}$ & -0.322 to -0.078 \\
\hline
\end{tabular}

Significant differences between group 1 and group 2 are shown in bold. 
Table 3 Mean central retina thickness (CRT) in $\mu \mathrm{m}$ for group 1 'recovery', group 2 'non-recovery' and their difference before and after 'upload' therapy and retreatment, respectively

\begin{tabular}{|c|c|c|c|c|}
\hline & $\begin{array}{l}\text { Mean CRT group } 1 \\
\text { 'recovery' }\end{array}$ & $\begin{array}{l}\text { Mean CRT group } 2 \\
\text { 'non-recovery' }\end{array}$ & $\begin{array}{l}\text { Difference } \\
\text { group 1-group } 2\end{array}$ & $\begin{array}{l}95 \% \mathrm{Cl} \text { of difference } \\
\text { group 1-group } 2\end{array}$ \\
\hline CRT before 'upload' therapy & $319.39 \pm 95.01$ & $279.09 \pm 79.55$ & $\begin{array}{l}-40.30 \pm 025.28 \\
p=0.123\end{array}$ & -92.18 to 11.58 \\
\hline CRT after 'upload' therapy & $215.67 \pm 70.75$ & $205.33 \pm 69.05$ & $\begin{array}{l}-10.33 \pm 20.01 \\
p=0.609\end{array}$ & -51.07 to 30.40 \\
\hline CRT before retreatment & $271.67 \pm 85.60$ & $281.03 \pm 89.39$ & $\begin{array}{l}9.36 \pm 24.74 \\
p=0.707\end{array}$ & -40.89 to 59.60 \\
\hline CRT after retreatment & $192.88 \pm 45.32$ & $213.84 \pm 66.09$ & $\begin{array}{l}20.97 \pm 16.28 \\
p=0.205\end{array}$ & -11.89 to 53.83 \\
\hline
\end{tabular}

line, ETDRS testing revealed an average decrease of 10 letters. It is known that VA testing using ETDRS charts is more sensitive than Snellen charts. ${ }^{24}$ However, it seems that this notion is even more pronounced in a routine clinical care situation. Insensitive VA testing may fail to identify patients with slowly decreasing VA in due course. In Germany, delay in retreatment due to formal processes in public health system has been shown to be associated with decreased functional recovery. ${ }^{25}$ This could be another reason for an 'undertreatment' of recurrent episodes in patients with nAMD, and it points out the necessity of a close cooperation between all involved parties: patients, referring ophthalmologists, treatment centre and public health system.

The fact that even though the OCT retreatment criteria of an 'increase of CRT of $100 \mu \mathrm{m}$ ' or more were still not fulfilled by all patients presenting with a decrease of VA of five letters or more may be due to the assumption that an increase of $100 \mu \mathrm{m}$ of CRT bears a ceiling effect. In addition, the analysis of a CRT change during upload therapy revealed a decrease of CRT of less than $100 \mu \mathrm{m}$ using an average of 3.6 injections. This observed change of CRT is less than in clinical trials such as CATT, ABC trial or PrONTO study. However, our results reflect the group of patients being treated in a clinical routine setting. Therefore, we interpret our results that quantitative OCT retreatment criteria (such as increase of CRT of $100 \mu \mathrm{m}$ or more) are not sensitive enough for early detection of recurrence of nAMD in a clinical routine setting. Instead, qualitative OCT criteria for retreatment such as intraretinal or subretinal fluid, as used in the CATT trial, should be used in daily clinical routine as they seem to depict intraretinal or subretinal changes faster and more sensitively than quantitative OCT-based retinal thickness measurements. ${ }^{11} 26$ This is also emphasised by the fact that almost all patients presenting with recurrence of nAMD showed some increase of CRT when compared with the end of upload therapy.

Dividing patients into 'recovery' and 'non-recovery' depending on whether they reach the same VA after treatment of their recurrence compared with their VA after initial 'upload' therapy showed that both subgroups did not differ in VA and CRT up until the end of the initial 'upload' therapy. VA after upload therapy and after retreatment differed significantly between groups 1 and 2 . Additionally, the functional retreatment parameter (decrease of VA five letters or more) was less often fulfilled in group 1 than group 2 . We interpret this subgroup analysis as an indicator for a weakness of the functional retreatment parameter in a more clinical setting. Recent recommendations include more detailed retreatment criteria such as the presence of any fluid or increase of any retinal thickness.

In the routine clinical care setting investigated, we failed to identify any other markers than 'drop of VA' to be of a predictive value for visual recovery after retreatment of nAMD.
Our study bears several weaknesses. First, it is a retrospective study with all known limitations. Additionally, we cannot comment on the outcome of all patients in the clinical setting investigated, as we only observed those who presented with a recurrence according to the then valid retreatment criteria. Although we believe this is the main reason for the clinical shortcoming of anti-VEGF treatment based upon these retreatment criteria, the results for all patients treated at that time may be even more behind expectations. However, as we intended to investigate a routine clinical care setting with referring ophthalmologists, this seems to be the most adequate study design for our main outcome variables.

Second, we cannot comment on other clinical setups in a single clinical centre with more uniform and standardised operational procedures. However, from our experience, the setting investigated in our current study is the most widely spread model for anti-VEGF therapy worldwide. In addition, the German public healthcare system does not cover newer treatment regimes such as 'treat and extend'.

Additionally, we did not evaluate quantitative findings such as fibrosis or atrophy. This was due to the retrospective nature of the study, which did not allow a standardised description or imaging during the follow-up.

As seen by the data during standardised 'upload' therapy, it seems that, in clinical routine, VA increase as well as CRT decrease differs from the observations in large prospective standardised clinical trials. However, for successful treatment of all patients, these data from routine clinical practice also have to be taken into account.

In summary, repeated intravitreal ranibizumab injections improve both VA and CRT of patients with naive and recurrent nAMD. In a routine clinical care setting, it seems that Snellen VA testing alone as a functional parameter is clearly not sufficient for the detection of early recurrence. Quantitative OCT retreatment criteria such as an increase of CRT of $100 \mu \mathrm{m}$ or more are also not sensitive enough to detect early recurrence in daily clinical routine. Instead, qualitative OCT-based retreatment criteria should be preferred. A further evaluation of more specific OCT retreatment criteria in a clinical setting is needed to provide and optimise treatment protocols for patients suffering from $\mathrm{nAMD}$.

Contributors All the authors were involved in: 1) substantial contributions to conception and design, acquisition of data, or analysis and interpretation of data; 2) drafting the article or revising it critically for important intellectual content; and 3) final approval of the version to be published.

Competing interests None.

Ethics approval Internal medical board.

Provenance and peer review Not commissioned; externally peer reviewed. 


\section{REFERENCES}

1 Kocur I, Resnikoff S. Visual impairment and blindness in Europe and their prevention. Br J Ophthalmol 2002;86:716-22.

2 Jager RD, Mieler WF, Miller JW. Age-related macular degeneration. N Engl J Med 2008;358:2606-17.

3 Algvere PV, Seregard S. Age-related maculopathy: pathogenetic features and new treatment modalities. Acta Ophthalmol Scand 2002;80:136-43.

4 Krzystolik MG, Afshari MA, Adamis AP, et al. Prevention of experimental choroidal neovascularization with intravitreal anti-vascular endothelial growth factor antibody fragment. Arch Ophthalmol 2002;120:338-46.

5 Avery RL, Pieramici DJ, Rabena MD, et al. Intravitreal bevacizumab (Avastin) for neovascular age-related macular degeneration. Ophthalmology 2006; 113:363-72.

6 Gonzales CR. Enhanced efficacy associated with early treatment of neovascular age-related macular degeneration with pegaptanib sodium: an exploratory analysis. Retina 2005;25:815-27.

7 Bressler NM, Chang TS, Suner IJ, et al. Vision-related function after ranibizumab treatment by better- or worse-seeing eye: clinical trial results from MARINA and ANCHOR. Ophthalmology 2010;117:747-56.

8 Cheung CM, Wong TY. Ranibizumab and bevacizumab for AMD. N Eng/ J Med 2011;365:2237.

9 Abraham P, Yue H, Wilson L. Randomized, double-masked, sham-controlled trial of ranibizumab for neovascular age-related macular degeneration: PIER study year 2 Am J Ophthalmol 2010;150:315-24.

10 Lalwani GA, Rosenfeld PJ, Fung $A E$, et al. A variable-dosing regimen with intravitreal ranibizumab for neovascular age-related macular degeneration: year 2 of the PrONTO Study. Am J Ophthalmol 2009;148:43-58.

11 Martin DF, Maguire MG, Ying GS, et al. Ranibizumab and bevacizumab for neovascular age-related macular degeneration. N Engl J Med 2011; 364:1897-908.

12 Schmidt-Erfurth U, Eldem B, Guymer R, et al. Efficacy and safety of monthly versus quarterly ranibizumab treatment in neovascular age-related macular degeneration: the EXCITE study. Ophthalmology 2011;118:831-9.

13 Holz FG, Amoaku W, Donate J, et al. Safety and efficacy of a flexible dosing regimen of ranibizumab in neovascular age-related macular degeneration: the SUSTAIN study. Ophthalmology 2011;118:663-71.

14 Singer MA, Awh CC, Sadda S, et al. HORIZON: An Open-Label Extension Trial of Ranibizumab for Choroidal Neovascularization Secondary to Age-Related Macular Degeneration. Ophthalmology 2012;119:1175-83.
15 Finger RP, Holz FG. [Access to healthcare services for elderly patients with neovascular age-related macular degeneration]. Ophthalmologe 2012;109:474-8

16 Framme C, Wolf-Schnurrbusch UE, Lobsiger $\mathrm{H}$, et al. [Adapted and standardised patient management in the treatment of neovascular AMD in the outpatient setting of a university eye hospital]. Klin Monb/ Augenheilkd 2012;229:812-21.

17 Wolf $A$, Reznicek $L$, Muhr J, et al. [Treatment of recurrent neovascular age-related macular degeneration with ranibizumab according to the PrONTO scheme.]. Ophthalmologe 2012;8:740-5.

18 Cohen SY, Dubois L, Ayrault S, et al. Ranibizumab for exudative AMD in a clinical setting: differences between 2007 and 2010. Graefe's archive for clinical and experimental ophthalmology=Albrecht von Graefes Archiv fur klinische und experimentelle. Ophthalmologie 2013;251:2499-503.

19 Colenbrander A, Fletcher DC. Low vision rehabilitation: visual acuity measurement in the low vision range. J Ophthalmic Nurs Technol 1992;11:62-9.

20 Bloch SB, la Cour M, Sander B, et al. Predictors of 1-year visual outcome in neovascular age-related macular degeneration following intravitreal ranibizumab treatment. Acta Ophthalmol 2013;91:42-7.

21 Brown DM, Regillo CD. Anti-VEGF agents in the treatment of neovascular age-related macular degeneration: applying clinical trial results to the treatment of everyday patients. Am J Ophthalmol 2007;144:627-37.

22 Patel PJ, Tufail A, for the ABCTI. Optimizing Individualized Therapy with Bevacizumab for Neovascular Age-Related Macular Degeneration. Retina Published Online First: 31 Jan 2012. doi:10.1097/IAE.0b013e31823f0ba3.

23 Wolf A, Kampik A. Efficacy of treatment with ranibizumab in patients with wet age-related macular degeneration in routine clinical care: data from the COMPASS health services research. Graefe's archive for clinical and experimental ophthalmology=Albrecht von Graefes Archiv fur klinische und experimentelle. Ophthalmologie 2014;252:647-55.

24 Cohen SY, Oubraham H, Uzzan J, et al. Causes of Unsuccessful Ranibizumab Treatment in Exudative Age-Related Macular Degeneration in Clinical Settings. Retina 2012;32:1480-5.

25 Muether PS, Hoerster R, Hermann MM, et al. Long-term effects of ranibizumab treatment delay in neovascular age-related macular degeneration. Graefe's archive for clinical and experimental ophthalmology=Albrecht von Graefes Archiv fur klinische und experimentelle. Ophthalmologie 2013;251:453-8.

26 Manousaridis K, Manjunath V, Talks J. Information used to decide on retreatment of exudative age-related macular degeneration with anti-VEGF in clinical practice. Eur $J$ Ophthalmol 2013;23:108-13. 


\section{Visual acuity and central retinal thickness: fulfilment of retreatment criteria for recurrent neovascular AMD in routine clinical care}

Lukas Reznicek, Johanna Muhr, Michael Ulbig, Anselm Kampik, Wolfgang J Mayer, Christos Haritoglou, Aljoscha Neubauer and Armin Wolf

Br J Ophthalmol 2014 98: 1333-1337 originally published online June 5, 2014

doi: 10.1136/bjophthalmol-2013-304399

Updated information and services can be found at:

http://bjo.bmj.com/content/98/10/1333

\section{These include:}

References This article cites 25 articles, 1 of which you can access for free at: http://bjo.bmj.com/content/98/10/1333\#BIBL

Email alerting Receive free email alerts when new articles cite this article. Sign up in the service box at the top right corner of the online article.

Topic Articles on similar topics can be found in the following collections Collections Retina (1608)

\section{Notes}

To request permissions go to:

http://group.bmj.com/group/rights-licensing/permissions

To order reprints go to:

http://journals.bmj.com/cgi/reprintform

To subscribe to BMJ go to:

http://group.bmj.com/subscribe/ 